\title{
Numerical Simulation and Characteristics Test of Temperature Field under Short-circuit Fault
}

\author{
Ying $\mathrm{Wu}^{1,2}$ \\ ${ }^{1}$ Shenyang Fire Research Institution, Ministry of Public Security, 110034, China \\ ${ }^{2}$ Key laboratory of fire scene investigation and evidence identification, Ministry of Public Security, 110034, China
}

\begin{abstract}
In this article, finite element software ANSYS was used to simulate the temperature field of short-circuit fault on copper conducting wire. A third-dimensional thermoelectric coupling model was established after analyzing the principle of shortcircuit fault. The problem of latent heat was solved by the enthalpy method. The paper simulated the changes of temperature field, so as to get vaporization volume of copper wires and calculate the splash of energy during transient short circuit, which are the most elements affecting on the splashing energy.
\end{abstract}

\section{Keywords-simulation; short-circuit fault; temperature field}

\section{INTRODUCTION}

Wire is used to connect electrical wiring and transfer electrical energy, which plays an important role in our daily life. Currently, with the development of the national economy and the improvement of people's living standards, the demand and use of electrical energy is increasing. Wires caused short circuit, electrical grounding, overload, poor contact and leakage of electricity by long-term run, part of electric line insulation aging and improper operation, which even led to electrical fires. Recently, the electrical fire in China both the cause of the fire and the direct economic loss of fire were all in the first place of the fire statistics. Electrical fire had been showing the situation of high and heavy losses major fires were often caused by electrical fire. Electrical fire had been a major causal factor in our society fire safety, causing huge casualties and property losses. Therefore, the study of wire's fault characteristics was particularly important.

During the actual work of electric wire/cable, due to the fact that line is subject to long-term external conditions such as dampness and corrosion, aging of wire/cable is accelerated, causing reduction of insulation performance. In case insulation is reduced to a certain degree, high field intensity generated by high voltage will cause breakdown of insulation material and finally occurrence of short-circuit fault; furthermore, unpredictable conditions such as artificial factor and natural force may also cause short-circuit accident of line, after occurrence of short-circuit fault, in case the instantaneous short-circuit current is beyond the setup scope of protection device, it will cause failure of protection device to actuate, meanwhile, instantaneous short-circuit current will cause metal granule to melt and splash, after the dropping on ambient combustible substance, it will cause combustion of combustible substance, therefore, short-circuit fault has extreme fire disaster hazard.
Due to short time duration of short-circuit, small volume of conductor contact area and uneven temperature distribution, it is still difficult to measure temperature distribution characteristics of conducting wire shorting area and adjacent medium through experiment at present, and it is only possible to analyze theoretically. Taking short-circuit caused by extrusion damage as an example, this article describes numerical simulation of short-circuit temperature, so as to provide theoretical criterion for research of electric short-circuit fire root-cause and its spread rule.

\section{ANALYSIS OF CONDUCTING Wire SHORT-Circuit PROCESS}

Figure 1 shows the actually measured short-circuit current and voltage waveform, conductor at short-circuit has experienced stages such as fusion, gasification and arc abrasion, etc, and two stages should be simulated respectively. In simulative calculation process, stage A to stage $\mathrm{C}$ are shortcircuit fusion and gasification stage, stage $\mathrm{C}$ to stage $\mathrm{D}$ are arc and extinction process. As per theoretical model, set initial structure parameter and material attribute of model, load shortcircuit current actually measured, calculate temperature field of two stages respectively, derive volume of metal fusion and gasification area, and compare with actually measured results, then correct model.

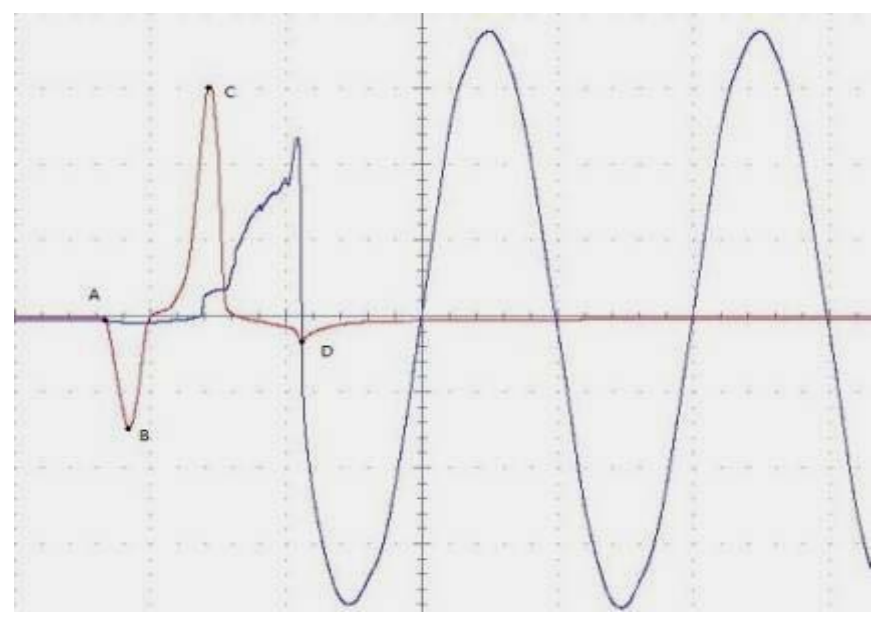

FIGURE I. ACTUALLY MEASURED SHORT-CIRCUIT CURRENT AND VOLTAGE WAVEFORM 


\section{NumERICAL SimUlation OF TEMPERATURE FiELD OF CONDUCTING WIRE SHORT-CIRCUIT}

\section{A. Establishment of Model}

Due to the fact that temperature of short-circuit point is very high in case of conducting wire short-circuit, its heat is transferred from conductive spot to other positions of conductor, and conductor temperature rise at position $0.01 \mathrm{~m}$ away from conductive spot can be ignored. In order to reduce computational complexity, take conductor of length $0.01 \mathrm{~m}$ for simulation. Figure 2 shows simulation model, length of both pieces of conductor is $0.01 \mathrm{~m}$, cross section area of conductor is $2.5 \mathrm{~mm} 2$, material of conductor is copper, the two conductors are parallel with each other and spacing is $0.02 \mathrm{~mm}$. Lap two conductors with cylinder, and its volume is to be determined.

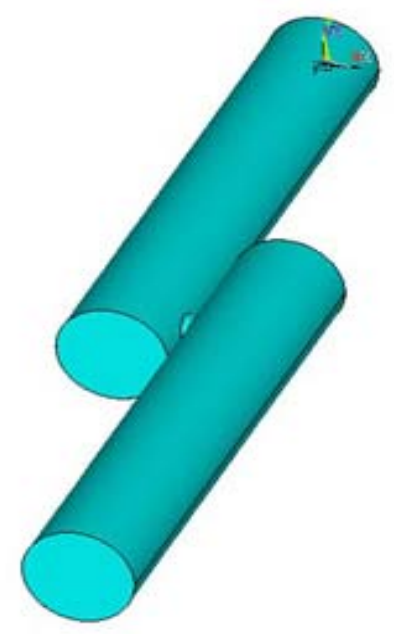

FIGURE II. SIMULATION MODEL OF CONDUCTING WIRE SHORTCIRCUIT

\section{B. Setup of Material Attribute}

Due to large temperature change of conductor material after conductor short-circuit, it is necessary to take account of the influence of temperature upon electrical conductivity and heat conductivity of copper.

After short-circuit of copper conductor, conductor material will be subject to fusion and gasification under high temperature, in order to take account of latent heat of phase change of material, adopt enthalpy method for handling of latent heat of phase change, introduce another physical quantity enthalpy, change of enthalpy value $\Delta H$ can be described as function of density, specific heat and temperature, and the following relationship exists:

$$
\Delta H=\int \rho c(T) \mathrm{d} T
$$

$\Delta H$ is the integration of product of density and specific heat to temperature, in unit of $\mathrm{J} / \mathrm{m} 3$.
ANSYS takes account of latent heat of phase change by defining enthalpy value of material with temperature change. Define latent heat of material into material enthalpy, enthalpy value changes with temperature, and enthalpy changes very rapidly in relation to temperature especially upon phase change. For pure material, difference between liquid temperature and solid temperature should be taken as very small temperature difference, so as to differentiate phase state through temperature interval.

Enthalpy curve can be divided into 3 zones as per temperature, substance is pure solid below solid temperature (Ts), is in phase change zone between solid temperature (Ts) and liquid temperature (T1), and is pure liquid above liquid temperature (T1), T0 is temperature of enthalpy value 0 . Calculate enthalpy values of temperature at all positions as per specific heat capacity and latent heat.

- Below solid temperature $\left({ }^{T<T_{\mathrm{s}}}\right)$

$$
H=\rho c_{\mathrm{s}}\left(T-T_{0}\right)
$$

In which, ${ }^{c_{\mathrm{s}}}$ is solid specific heat capacity.

- At solid temperature $\left({ }^{T}=T_{\mathrm{s}}\right)$

$$
H_{\mathrm{s}}=\rho c_{\mathrm{s}}\left(T_{\mathrm{s}}-T_{0}\right)
$$

- Between solid temperature and liquid temperature (phase change area) $\left(T_{\mathrm{s}}<T_{1}\right)$

$$
\begin{aligned}
& H=H_{\mathrm{s}}+\rho c^{*}\left(T-T_{\mathrm{s}}\right) \\
& C_{\text {avy }}=\frac{\left(C_{\mathrm{s}}+C_{1}\right)}{2} \\
& C^{*}=C_{\text {avy }}+\frac{L}{\left(T_{1}-T_{\mathrm{s}}\right)}
\end{aligned}
$$

In which, ${ }^{c_{1}}$ is liquid specific heat capacity, and $L$ is latent heat of fusion

- $\quad$ At liquid temperature $\left(T=T_{1}\right)$

$$
H_{1}=H_{\mathrm{s}}+\rho c^{*}\left(T_{1}-T_{\mathrm{s}}\right)
$$

- $\quad$ Over liquid temperature $\left(T_{1}<T\right)$

$$
H=H_{1}+\rho c_{1}\left(T-T_{1}\right)
$$

As per analysis above, the issue of latent heat has been transformed as enthalpy value, and different enthalpy values are taken in calculation process when fusion body is at different temperature stages. Physical parameters of copper material are shown in table 1 , and the calculated enthalpy values are shown in table 2 . 
TABLE I. PHYSICAL PARAMETERS OF COPPER MATERIAL

\begin{tabular}{|l|l|}
\hline \multicolumn{1}{|c|}{ Material Physics Character Parameters } & \multicolumn{1}{|c|}{ Numerical Value } \\
\hline Melting point $/{ }^{\circ} \mathrm{C}$ & 1083 \\
\hline Boiling point $/{ }^{\circ} \mathrm{C}$ & 2595 \\
\hline $\mathrm{\rho} /(\mathrm{kg} / \mathrm{m} 3)$ & 8900 \\
\hline $\mathrm{Cs} /[\mathrm{J} /(\mathrm{kg} \cdot \mathrm{K})]$ & 380 \\
\hline $\mathrm{Cl} /[\mathrm{J} /(\mathrm{kg} \cdot \mathrm{K})]$ & 522 \\
\hline $\mathrm{Cg} /[\mathrm{J} /(\mathrm{kg} \cdot \mathrm{K})]$ & 596 \\
\hline Melting heat $/(\mathrm{J} / \mathrm{kg})$ & $2.034 \times 105$ \\
\hline Boiling heat $/(\mathrm{J} / \mathrm{kg})$ & $4.763 \times 106$ \\
\hline
\end{tabular}

TABLE II. ENTHAPLY OF COPPER UNDER DIFFERENT EMPERATURES

\begin{tabular}{|l|l|}
\hline \multicolumn{1}{|c|}{ Temperature $^{\circ}{ }^{\circ} \mathrm{C}$} & enthaply/ $(\mathrm{J} / \mathbf{m} 3)$ \\
\hline 20 & 0 \\
\hline 1072 & $3.5579 \times 109$ \\
\hline 1084 & $2.5329 \times 1010$ \\
\hline 2586 & $2.7250 \times 1012$ \\
\hline 2596 & $3.1490 \times 1012$ \\
\hline 2900 & $3.1654 \times 1012$ \\
\hline
\end{tabular}

Actual contact area of metal is reduced due to unevenness of contact area. Therefore, when electric current flows through conductor, serious constriction phenomenon of current flow line occurs near contact area, i.e. effective conduction cross section area of conductor near contact area is reduced substantially, causing increase of resistance, i.e. the action of constriction resistance. Meanwhile, a layer of oxide film of poor electric conductivity is quickly formed in air and attached on surface of contact area, increasing resistance, this portion of resistance is called membrane resistance. Therefore, contact resistance consists of constriction resistance and membrane resistance. When current flows through contact position between two conductors, due to existence of contact resistance at contact position, local high temperature phenomenon occurs.

As temperature of short-circuit point rises rapidly in shortcircuit process and short-circuit point will be oxidized, therefore, lapping point adopts copper oxide material. Table 3 is the material attribute table of copper oxide.

TABLE III. MATERIAL ATTRIBUTE OF COPPER OXIDE

\begin{tabular}{|l|l|l|l|l|}
\hline $\begin{array}{c}\text { Material } \\
\text { attribute }\end{array}$ & $\begin{array}{c}\text { density } \\
\left(\mathrm{kg} / \mathrm{m}^{3}\right)\end{array}$ & $\begin{array}{c}\text { resistivi } \\
\text { ty } \\
(\Omega \cdot \cdot m\end{array}$ & $\begin{array}{c}\text { specific heat } \\
\text { capacity } \\
\left(\mathbf{J} / \mathbf{k g} .{ }^{\circ} \mathrm{C}\right)\end{array}$ & $\begin{array}{c}\text { coefficient of } \\
\text { thermal } \\
\text { conductivity } \\
\text { (W/m.k) }\end{array}$ \\
\hline $\mathrm{CuO}$ & 6500 & 0.0014 & 655 & 80 \\
\hline
\end{tabular}

\section{Element Selection}

This article researches thermoelectric coupling process of conducting wire short-circuit, therefore, selects physical element of SOLID69, which has two degrees of freedom of voltage and temperature, as a 3-dimension, 8-node and hexahedron element, it can be used for transient and stable thermoelectric coupling analysis and description of the process of generation of Joule heat through electric current, and it covers thermal equilibrium process and meets calculation demand of model well.

\section{Grid Division}

The grid division directly determines whether the finite element calculation results of ANSYS is correct or not, and affects accuracy of solution and speed of calculation. Grid division is generally classified into three methods: free grid division, mapping grid division and sweeping grid division. Here model becomes irregular due to curved surface contact between cubic lapping point and conducting wire, therefore, model is divided into tetrahedral elements by adopting free grid division, then grid size and spacing distribution is automatically controlled by intelligent size control technology (SMARTSIZE command) of ANSYS.

\section{E. Setup of Analysis Type and Load}

During short-circuit of conducting wire, heat source current is changing, and resistivity, specific heat capacity and thermal conductivity of material are changing too, therefore, thermoelectric coupling process of conducting wire requires transient analysis, and temperature field at any instance can be calculated.

Temperature rise of conducting wire is due to Joule heat generated by current, therefore, the load here is current, loading of current is to apply current vector by selecting a node on a end face of conducting wire and perpendicular to the end face, current size is the data value derived from experiment and coupled with degree of freedom of voltage, so that the potential at the cross section is equivalent, i.e. current density is distributed evenly; select all nodes on the other end face, define voltage as zero constantly, i.e. define reference plane of zero potential; initial condition of temperature field of model is environmental temperature $20^{\circ} \mathrm{C}$.

\section{F. Loading and Derivation}

Realize loading of alternative current by adopting macro command. Time step length of experimental data is $0.0001 \mathrm{~s}$, it is possible to increase step length appropriately as per demand of computational complexity so as to optimize calculation. Derive element temperature of model and corresponding element volume data table, calculate gasification volume and size of splashing energy.

\section{Simulation Results}

Figure 3 shows temperature distribution cloud chart of conducting wire cross section $6.6 \mathrm{e}-7 \mathrm{~m} 2$ and oxide film thickness $0.0002 \mathrm{~m}$. In which, (a) is temperature distribution cloud chart and close-up view at the starting instance of conducting wire short-circuit, the instance corresponds to point $\mathrm{A}$ in figure 1 , it can be seen that temperature of short-circuit point at the instance is higher than temperature of other positions. Figure (b) is temperature distribution cloud chart corresponding to point $\mathrm{B}$ in figure 1 , current at the instance has reached the 1 st peak value of $237 \mathrm{~A}$, while conducting wire just starts to melt down, as melting point of copper is $1083^{\circ} \mathrm{C}$, find out all elements higher than $083^{\circ} \mathrm{C}$ and sum up corresponding element volume, so as to get the fusion volume $1.710 \mathrm{e}-11 \mathrm{~m} 3$. Figure (c) shows the temperature distribution at the final instance, i.e. corresponding to point $\mathrm{C}$ in figure 1 , the current value at the instance is $-925 \mathrm{~A}$, temperature of conducting wire 
short-circuit point reaches the highest temperature, and conducting wire short-circuit point has gasified, the volume of gasification is $1.6423 \mathrm{e}-12 \mathrm{~m} 3$. In accordance with similar method, as gasification point of copper is $2585^{\circ} \mathrm{C}$, find out all elements which are higher than gasification point and sum up their volume, and get fusion volume of $9.564 \mathrm{e}-11 \mathrm{~m} 3$.

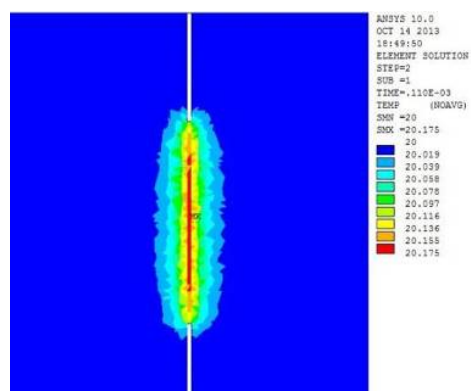

a) point $A$

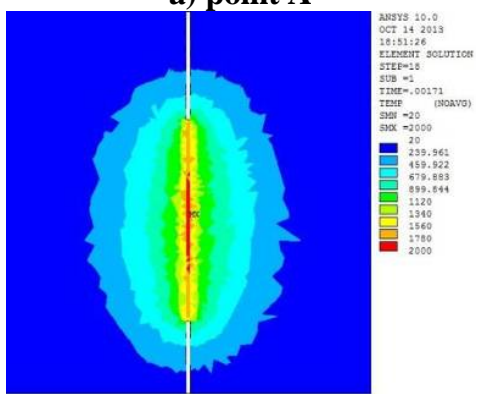

b) point $B$

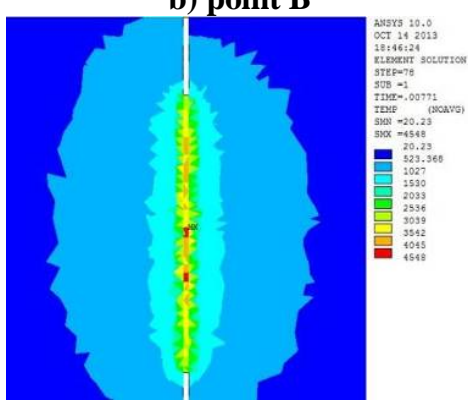

c) point $\mathrm{C}$

FIGURE III. TEMPERATURE DISTRIBUTION CLOUD CHART

\section{SUMMARY}

Through simulation and test of temperature distribution of electric short-circuit malfunction, the technical problem of difficulty to measure temperature distribution characteristics of shorting fusion (gasification) zone and adjacent medium has been solved firstly, so that technical criterion has been provided for electric circuit fire prevention and root-cause analysis.

\section{REFERENCES}

[1] Zhang Jinzhuan and Jianghao. Fire Safety Science, Vol. 17, 2008, p. 63.

[2] Mo Shanjun, Peng Wenjin. Journal of Safety Science and Technology, Vol. 8, 2012, p. 63

[3] Wang Fuchi and Zhang Chaohui: Theory and Engineering Example of ANSYS10.0 Finite Element Analysis (Beijing: Publishing House of Electronics Industry, 2006).

[4] Cao Yundong, Electric Theory (China Machine Press, 2012)
[5] Zhang Guozhi, Hu Renxi and Chen Jigang. Guidance Teaching Course of ANSYS10.0 Thermodynamics Finite Element Analysis (Beijing: China Machine Press, 2007). 